\title{
FDG-PETCT versus contrast-enhanced computed tomography in diagnosis of post-therapeutic colorectal cancer recurrence and metastases
}

\author{
Nivine Abdel Moneim Chalabi ${ }^{1}$, Reem Hassan Bassiouny ${ }^{1 *}$ (D) and Mohamed Abobakr El Sedek ${ }^{2}$
}

\begin{abstract}
Background: This study was designed to assess the role of 18F-FDG CT was able to detect additional/CT in posttherapeutic surveillance of colorectal cancer patients as compared with contrast-enhanced CT to allow early detection of recurrent and metastatic cases amenable for curative surgery and thus improve the overall survival of patients.

Results: Of the total 100 patients, 70 proved to have metastasis or local recurrent disease by the standard reference modalities. One hundred eighty-two diseased regions were detected in these 70 patients. PET/CT was positive in 174 regions (95.6\%) whereas CECT was positive in 118 regions (64.8\%). PET/CT was superior to CECT in detection of hepatic focal lesions, metastatic lymph nodes, pulmonary metastases, and peritoneal and suprarenal metastases whereas both were equal in detection of osseous deposits. CECT detected four lesions that were missed by PET/CT, and these were hepatic metastases from mucinous adenocarcinoma.

Conclusion: PET/CT is a better method to evaluate post-therapeutic colorectal cancer patients. It detected viable residual tumor cells in operative bed scar, small LNs, hepatic focal lesions, peritoneal deposits, pulmonary masses, bone deposits, and suprarenal deposits with significantly higher specificity and sensitivity than CECT avoiding unnecessary surgeries.
\end{abstract}

Keywords: PET/CT, Recurrent and metastatic colorectal cancer

\section{Background}

Colorectal cancer (CRC) is the third most commonly diagnosed cancer in males and the second in females, and is one of the leading causes of cancer-related morbidity and mortality that accounted for almost 835,000 deaths in 2015 [1]. Despite the fact that radical surgery is curative in up to $70 \%$ of colorectal cancer patients, $30-50 \%$ of them will still develop post-operative locoregional recurrence or metastasis within 2 years of primary surgery with or without adjuvant chemotherapy and will die with their disease [2, 3]. However, a good proportion of patients with recurrent or metastatic CRC still have the chance of curative-intent surgery which is associated

\footnotetext{
* Correspondence: rhbassiouny1592@gmail.com

'Ain Shams University, Radiodiagnosis Department, Cairo, Egypt Full list of author information is available at the end of the article
}

with a 5 -year survival rate of $30-40 \%$ in selected patient populations with single organ metastasis [4]. The principal aim of follow-up programs after radical resection of colorectal cancer is to allow early detection of the recurrence and metastasis in order to enhance the proportion of potentially resectable cases and thus improve the prognosis and survival of patients [5]. The postoperative follow-up of CRC patients includes regular physical examination, estimation of the serum carcinoembryonic antigen (CEA) level, endoscopy, and conventional imaging techniques such as contrast-enhanced computerized tomography (CECT), magnetic resonance imaging (MRI) and ultrasound of the liver [4]. Elevated CEA values cannot provide accurate localization to the sites of recurrence [6]. CT has been considered the primary imaging investigation for detection of recurrent 
CRC because of its low cost, widespread availability, and high resolution of anatomic details; however, it cannot discriminate recurrent neoplastic disease from nonmalignant changes, such as scars, inflammation, and radiation necrosis and may under-estimate the actual tumor burden by overlooking small tumor clusters in areas of distorted anatomy after treatment [7]. 18 Fluorine (18F) fluorodeoxyglucose (FDG) positron emission tomography $(\mathrm{PET} / \mathrm{CT})$ is an integrated imaging modality of anatomic and functional imaging that can readily detect the cancer-related metabolic changes which usually precede the morphological ones $[8,9]$. It was considered by many authors to be more sensitive than the conventional imaging modalities in the early detection of local recurrence and distant metastases and in evaluation of tumor viability following radiotherapy and chemotherapy in colorectal patients with elevated or normal CEA $[2,10]$. However, the diagnostic value and efficacy of FDG PET/ CT in surveillance of CRC patients are not yet fully established [11].

In this prospective study, our goal was to assess the role of 18F-FDG PET/CT in comparison with enhanced $\mathrm{CT}$ in post-therapeutic surveillance of CRC patients and its sensitivity for early detection of post-therapeutic colorectal cancer recurrence and metastasis

\section{Methods}

\section{Patients}

This prospective study included 100 patients with histopathologically proven colorectal cancer who were referred to perform post-therapeutic follow-up by PET/CT in the period between January 2014 and January 2018 . The study took place in an Egyptian military hospital and was approved by its ethical committee. The inclusion criteria were as follows: (1) histopathologic confirmation of primary CRC including TMN classification; (2) curative resection of the tumor either or not followed by chemoradiation therapy; (3) regular follow-up program every 3 or 6 months, including physical examinations, serum CEA level estimation, and conventional imaging including ultrasound of the liver and CT chest and pelviabdomen; (4) patients with suspected local recurrence or metastasis by routine follow-up program. (5) No age predilection and both sexes were included. Exclusion criteria included patients with strong history of atopic disorders, and those with serum creatinine level above 2 $\mathrm{mg} / \mathrm{dl}$, recent surgery less than 6 weeks, radiotherapy within less than 3 months, or chemotherapy within less than 3 weeks.

\section{Technique}

PET/CT was performed on an integrated scanner (Philips 128 slice $\mathrm{CT}$ ) that combines both $\mathrm{CT}$ and PET
Table 1 Patient characteristics

\begin{tabular}{ll}
\hline Total number of patients & 100 \\
\hline Male patients & 54 \\
Female patients & 46 \\
Mean age for males (age range from 40 to 76) years & 59.9 \\
Mean age for females (age range 26-76) years & 56.8 \\
\hline
\end{tabular}

capabilities in two sequential gantries, to avoid the need to move the patient between the two components of the study. Patients fasted for at least $6 \mathrm{~h}$ before the examination, except for water. Blood glucose levels were measured and should be less than $200 \mathrm{mg} / \mathrm{dl}$. An intravenous dose of $(0.18-0.21 \mathrm{mCi} / \mathrm{kg}, 5-14 \mathrm{mCi})$ FDG was injected and after 45-60 min; the patients were asked to void just before entering the examination room. Multi-detector CT examination from the base of the skull to the upper thighs was planned with the following parameters: 120 $\mathrm{mA}, 140 \mathrm{kVp}$, table speed $=13.5 \mathrm{~mm}$ per rotation, and thickness of $4 \mathrm{~mm}$. After CT acquisition, PET scan of the same axial range started with the patient in the same position on the table for $2-3 \mathrm{~min}$ per bed position. PET data were acquired by using a matrix of $128 \times 128$ pixels. CT-based attenuation correction of the emission images was used. Contrast-enhanced CT was performed by the same scanner $20-50 \mathrm{~s}$ after giving bolus injection of non-ionic iodinated contrast at dose about $2-3 \mathrm{ml} / \mathrm{kg}$ of body weight. Scanning was acquired from the base of the skull till the mid-thigh and may involve the whole body in case of extensive skeletal deposits, using the 2.5mm thickness section.

\section{Image interpretation}

The reconstructed attenuation corrected PET images, CT images, and fused images of matching pairs of PET and CT images were reviewed by two experienced nuclear medicine physicians and a radiologist in axial, coronal, and sagittal planes, as well as in maximum intensity projections and in three-dimensional cine mode. Qualitative assessments for the presence of hyper-metabolic lesions were evaluated on

Table $\mathbf{2}$ Local recurrences or metastases proved by reference modalities

\begin{tabular}{llll}
\hline Region & Number of regions & PET/CT & CECT \\
\hline Local recurrence & 38 & $38(100 \%)$ & $28(73.68 \%)$ \\
Hepatic deposits & 52 & $48(92.3 \%)$ & $40(76.9 \%)$ \\
Metastatic lymph nodes & 40 & $40(100 \%)$ & $12(30 \%)$ \\
Pulmonary metastasis & 28 & $24(85.7 \%)$ & $22(78.8 \%)$ \\
Peritoneal deposits & 16 & $16(100 \%)$ & $10(62.5 \%)$ \\
Suprarenal metastasis & 2 & $2(100 \%)$ & $2(100 \%)$ \\
Osseous deposits & 6 & $6(100 \%)$ & $4(66.6 \%)$ \\
Total & 182 & $174(95.6 \%)$ & $118(64.8 \%)$ \\
\hline
\end{tabular}



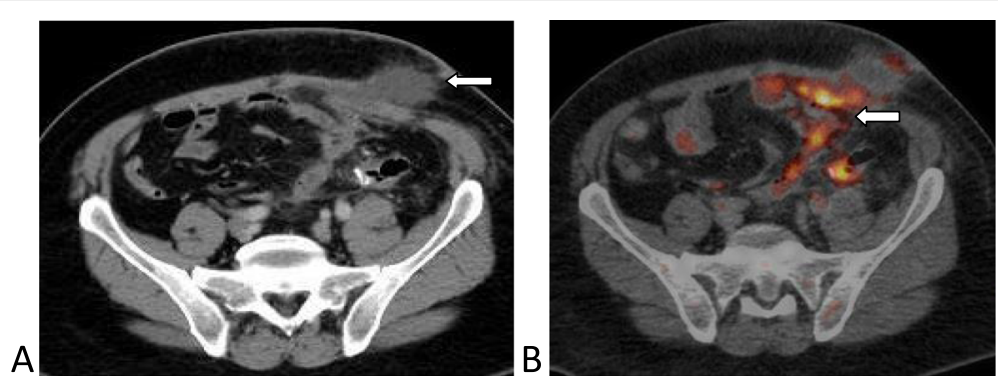

Fig. 1 A 52-year-old male patient who underwent surgical resection for pathologically proven colonic adenocarcinoma followed by chemotherapy and radiotherapy and follow-up 6 months later revealed a normal tumor marker level. a Axial post-contrast CT image showing operative bed fluid collection (seroma) with no enhancing component (arrow), b axial fused PET-CT image showing avid metabolic activity at the operative bed

corrected PET images. Semi-quantitative evaluation was performed using the standardized uptake value (SUVmax). Lesions which exceeded SUVmax of 2.5 were considered positive for disease involvement whereas findings with SUVmax below 2.5 were considered insignificant. Contrastenhanced CT images were evaluated for the presence of hepatic focal lesions, lymph node size (more than $10 \mathrm{~mm}$ in its short axis), lymph node morphology, pulmonary nodules, peritoneal masses, operative bed masses, and skeletal lesions. Comparison with other clinical and diagnostic methods including laboratory tests (tumor markers) and other pathological findings was performed.

\section{Reference standard}

Our reference standard included histopathological or cytological confirmation or at least 6 months of clinical and radiological follow-up to confirm the final diagnosis of recurrence or metastasis.

\section{Statistical analysis}

IBM SPSS statistics (V. 23.0, IBM Corp., USA, 2015) was used for data analysis. Diagnostic validity test was used: it includes agreement and disagreement between PET/ $\mathrm{CT}$ and CECT. The Chi-square test was used to compare the differences between the two studied imaging modalities. The probability of error equal 0.05 was considered significant; while values at 0.01 and 0.001 are highly significant. PET/CT findings and CECT findings were classified as true positive (TP), false positive (FP), true negative (TN), and false negative (FN), as compared with those of the reference standard. Standard statistical formulas were used to calculate the sensitivity, specificity, and accuracy of 18F-FDG PET/CT and CECT in both the case-based and lesion-based analyses.

\section{Results}

The sociodemographic variables and lesion characteristics of the 100 patients included in the study are shown in Table 1 . Seventy out of the hundred patients $(70 \%)$ proved to have metastasis or local recurrent disease by the standard reference modalities. Forty-two patients were confirmed by histopathology while 28 patients by follow-up regimes. One hundred eighty-two diseased regions were detected in these 70 patients with distribution as shown in Table 2. By comparing the results of 18F-FDG PET/CT and CECT (Table 2), we found that 18F-FDG PET/CT was superior to CECT by detection of all 38 local recurrent lesions (100\%) compared with only 28 (73.6\%) detected by CECT (e.g., Figs. 1 and 2). PET/ CT accurately detected 48 out of the 52 hepatic focal

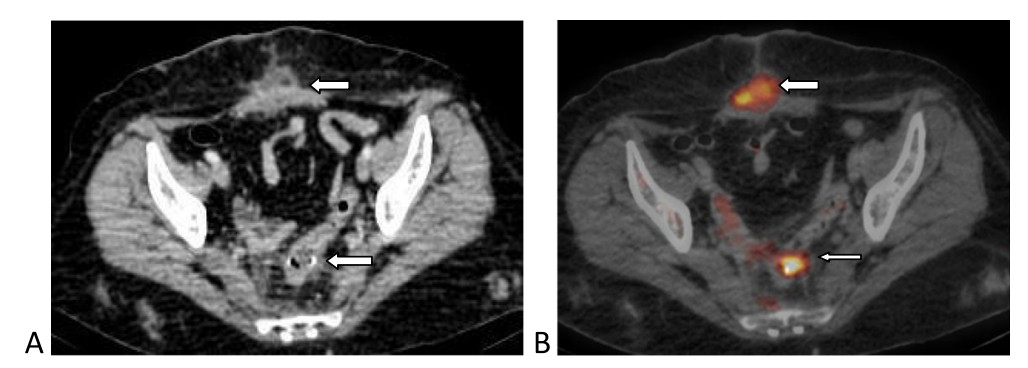

Fig. 2 A 64-year-old female patient who underwent surgical resection for pathologically proven colonic adenocarcinoma, presented with an elevated tumor marker. a Axial post-contrast $C T$ image showing early post-operative enhancing scar and minimal thickening at the anastomotic site (arrows), $\mathbf{b}$ axial fused PET-CT image showing corresponding metabolic activity in the base line PET-CT (arrows) 


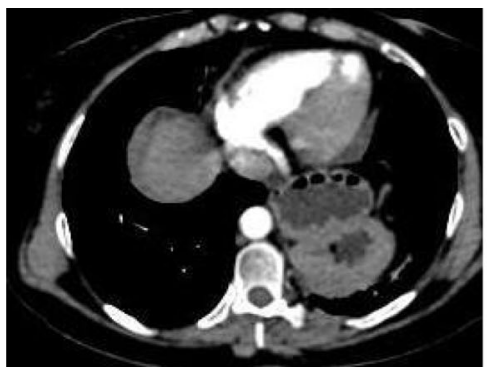

A

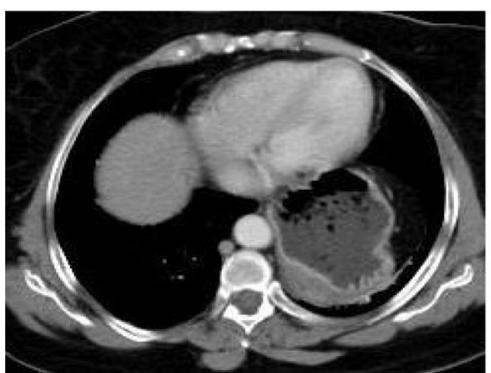

C

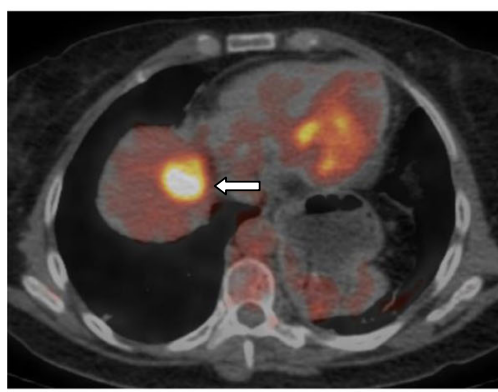

E

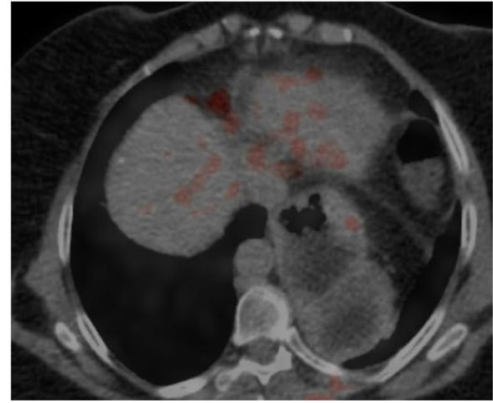

G

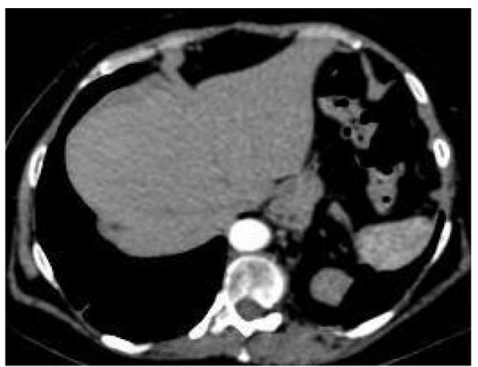

B

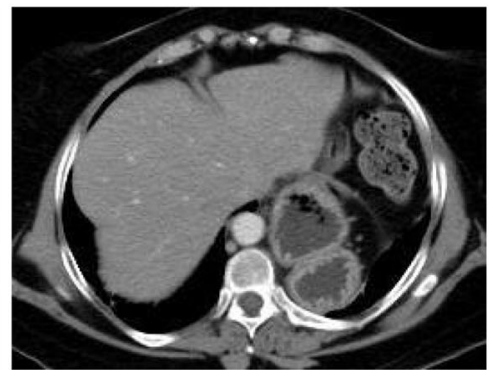

D

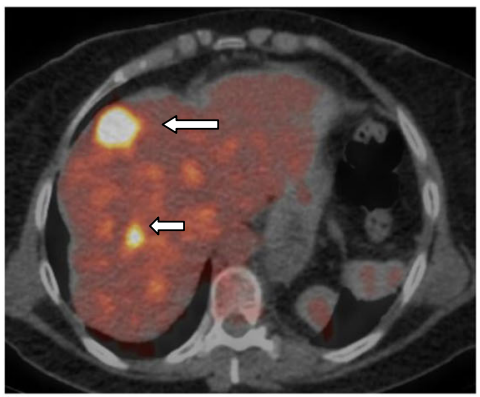

$\mathrm{F}$

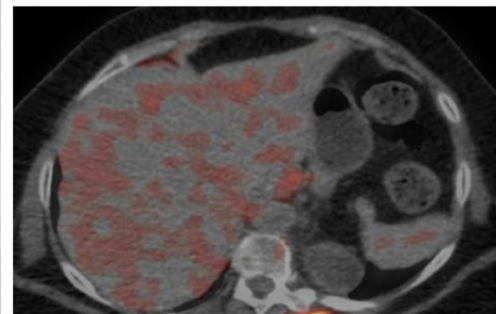

$\mathrm{H}$

Fig. 3 A 64-year-old female patient who underwent surgical resection for pathologically proven colonic adenocarcinoma, presented with an elevated tumor marker. $\mathbf{a}$, $\mathbf{b}$ Axial post-contrast $C T$ images in arterial phase showing no enhancing focal lesion. $\mathbf{c}$, $\mathbf{d}$ Axial post-contrast $C T$ images in porto venous phase showing no enhancing focal lesion. $\mathbf{e}, \mathbf{f}, \mathbf{d}$ Axial fused PET-CT images at the same level of the enhanced CT images showing metabolically active hepatic focal lesions (arrows). $\mathbf{g}$, $\mathbf{h}$ Post-therapeutic axial fused PET-CT images showed good therapeutic response with disappearance of the metabolically active hepatic focal lesions

lesions (92.3\%) and CECT detected 40 lesions (76.9\%). $\mathrm{PET} / \mathrm{CT}$ was able to detect additional 8 hepatic deposits, not detected by CT (e.g., Fig. 3), while CECT detected 4 lesions that were missed by PET/CT and were hepatic metastases from mucinous adenocarcinoma (e.g., Fig. 4).
PET/CT detected all 40 metastatic lymph nodes (100\%), while the CECT detected only 12 (30\%). PET/CT accurately detected 24 out of the 28 pulmonary metastases (85.7\%) whereas CECT detected only 22 (78.8\%) (e.g., Fig. 6). PET/CT accurately detected other lesions 

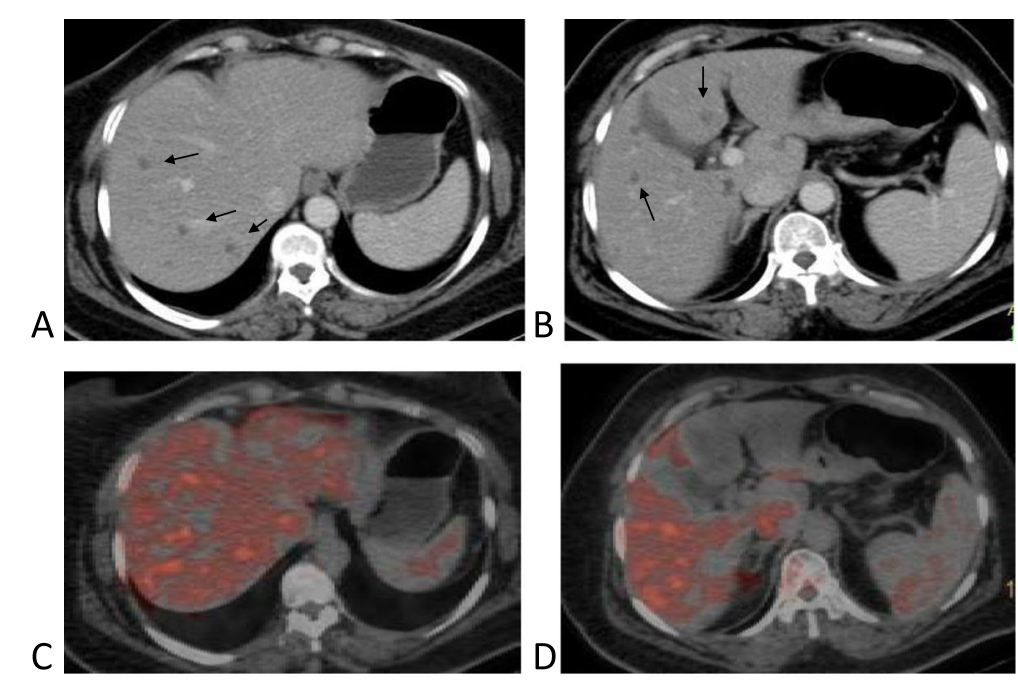

Fig. 4 A 40-year-old male patient who underwent rectal resection for pathologically proven mucinous adenocarcinoma, not followed by chemo or radiotherapy. Follow-up revealed elevated level of tumor markers. $\mathbf{a}$, b Axial post-contrast $\mathrm{CT}$ images showing multiple small sized hypodense non-enhancing hepatic focal lesions (arrows). c, d Axial fused PET/CT images showing no avid metabolic hepatic focal lesions at the same levels which proved to be metastatic deposits

including (16 peritoneal, 2 suprarenal, and 6 osseous deposits, e.g., Fig. 7), and CECT detected 19 lesions only (10 peritoneal, 2 suprarenal, and 4 osseous deposits) as shown in Table 3. In the assessment of the 100 patients 252 sites of recurrent sites were suspected by both PET/ CT and CECT. PET-CT agreed with reference modalities in 238 lesions as it was true positive in detection of 174 lesions and true negative in detection of 64 regions.
PET-CT disagreed with reference modalities in 14 lesions (5.8\%) as it was false negative in 8 lesions (two hepatic and two pulmonary) and false positive in 6 operative bed recurrence regions. The level of agreement between the results of both PETCT and reference modalities was about $94.4 \%$ with $p$ value $=0.000$ which is highly significant (Table 3). CECT agreed with reference modalities in 148 lesions as it was true positive in detection

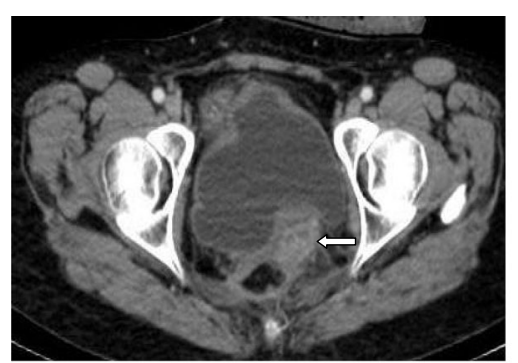

A

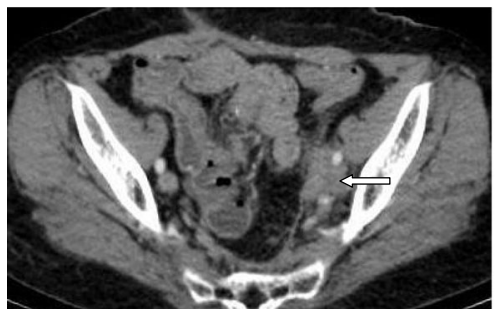

C

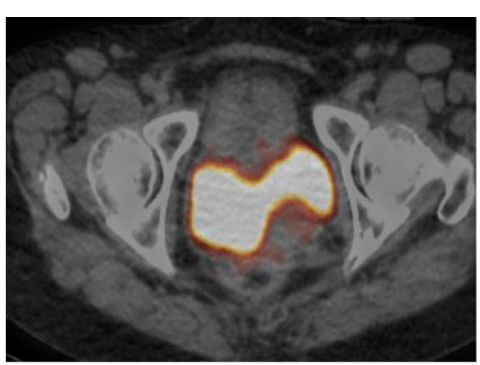

B

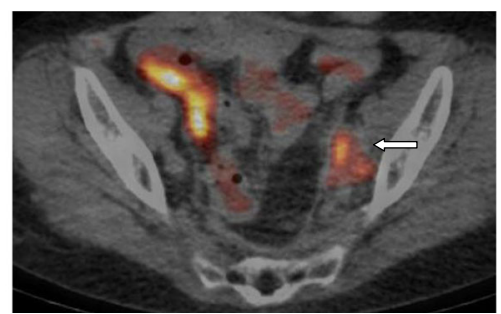

D

Fig. 5 A 57-year-old female patient who underwent rectal resection for pathologically proven adenocarcinoma, followed by chemotherapy, presented 4 months later by left loin pain and elevated tumor markers. a Axial post-contrast CT image showing operative bed faintly enhancing mass (arrow) encasing the distal left ureter (arrow). b Axial fused PET-CT image showing no metabolically active lesions noted in the operative bed. c Axial post-contrast CT image showing left iliac enlarged LNs (arrow). d Axial fused PET-CT image showing left iliac metabolically active LNs (arrow) 


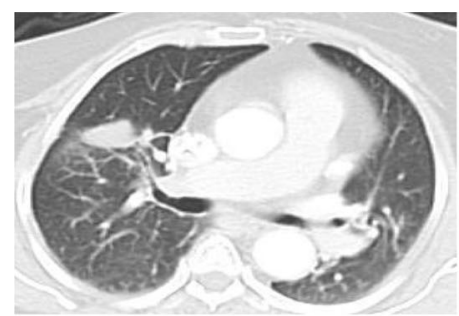

A
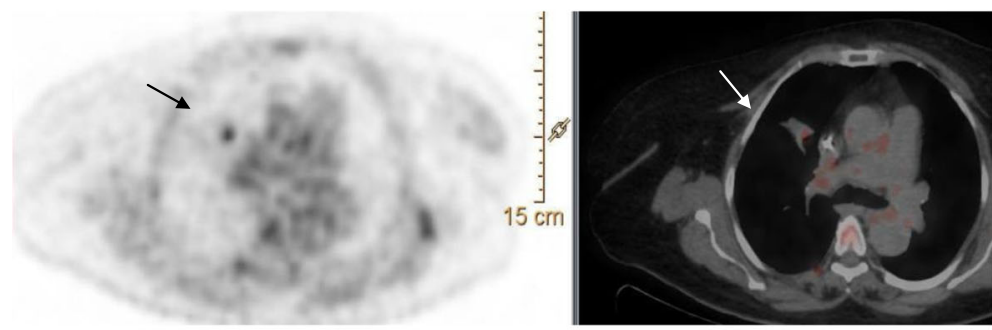

B

C

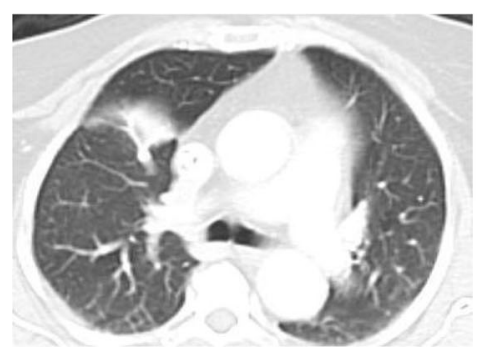

D
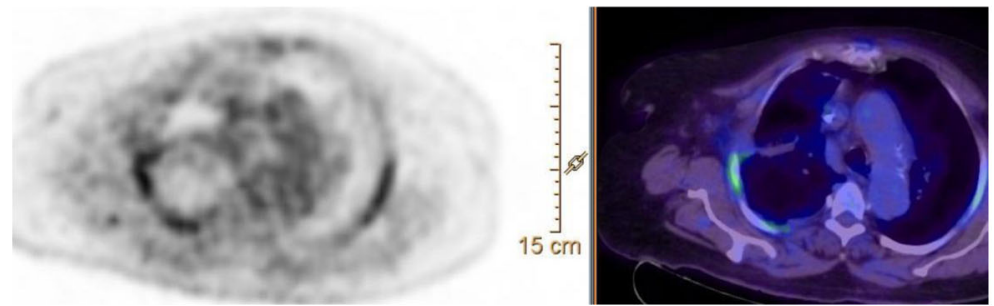

Fig. 6 A 70-year-old female patient who underwent colonic mass surgical resection for pathologically proven adenocarcinoma, presented 4 months later by elevated tumor markers. a Axial CT image in lung window showing right upper lobe pulmonary nodule. b Axial PET attenuated corrected image and axial fused PET-CT image of right upper lobe metabolically active nodule (arrows). c Post-radiofrequency ablation assessment for the pulmonary nodule by contrast-enhanced $C T$ showed stationary size depending on the size criteria (appreciable change in size needs time). $\mathbf{d}$ PET-CT revealed a photopenic area corresponding to the ablated nodule with no metabolically active lesion within

of 114 lesions and true negative in 34 lesions, yet CECT disagreed with reference modalities as it was false negative (Fig. 6), false positive (Fig. 5) as shown in Table 4 and figure. The agreement between the results of both CECT and reference modalities was about $58.7 \%$ and $p$ value $=0.51$ which is insignificant. The diagnostic performance of PET/ $\mathrm{CT}$ and CECT in the detection of locoregional recurrence and metastases is shown in Table 5 with higher sensitivity, specificity, positive predictive value, negative predictive value, and diagnostic efficacy of PET/CT as compared with enhanced CT. The sensitivity and accuracy of FDG PET/
CT significantly differed from those of CECT in lesionbased analyses $(p<0.05)$, whereas for the specificity, the $\mathrm{PET} / \mathrm{CT}$ results did not show a significant difference from those of CECT.

\section{Discussion}

Although contrast-enhanced computed tomography is considered the primary imaging modality in the detection of local recurrence in patients with colorectal cancer, its limitations include its reliance on size criteria in the diagnosis of metastatic LNs, enhancing 


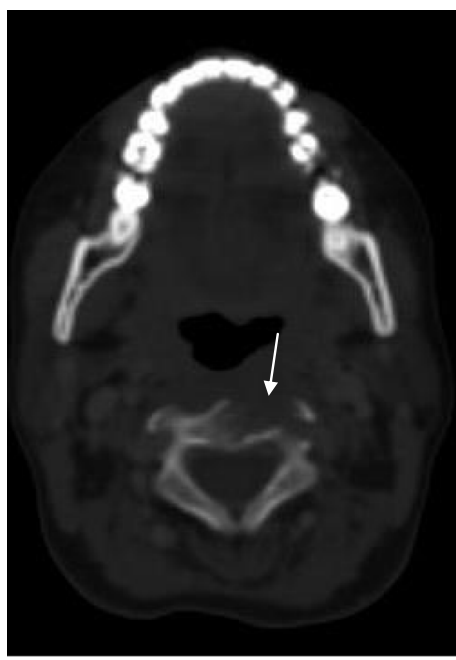

A

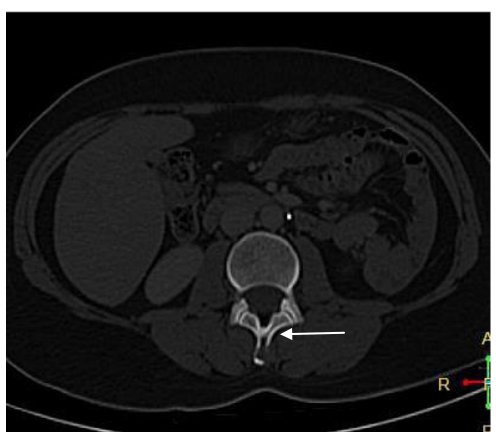

C

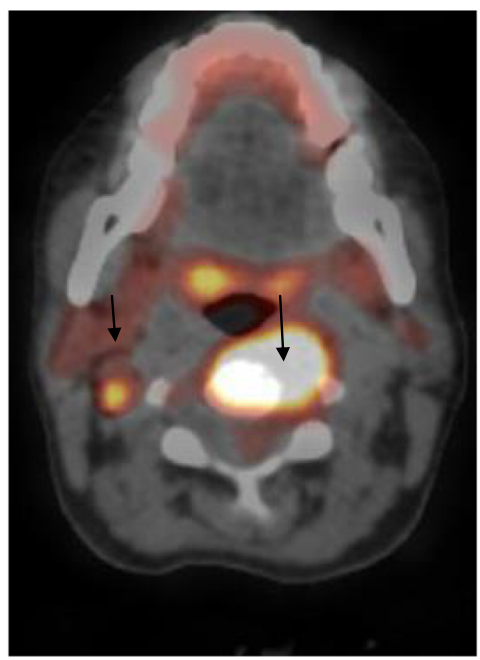

B

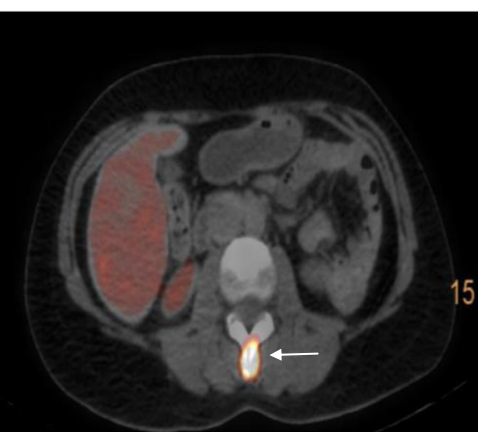

D

Fig. 7 A 38-year-old female patient who underwent rectal surgical resection for pathologically proven adenocarcinoma, not followed by chemo or radiotherapy. Recently, she presented with neck pain, low back pain, and elevated tumor markers level. a Axial CT image in bone window showing destructive cervical vertebral body lesion (arrow). b axial fused PET-CT image at the same level showing metabolically active lesion destroying the vertebral body of C2 as well as avid right deep cervical LN (arrows). c Axial CT image in bone window showing a small osteolytic lesion in the spinous process of the second lumbar vertebra (arrow). $\mathbf{d}$ Axial fused PET-CT image at the same level showing metabolic activity within the lesion of the spinous process of the second lumbar vertebra (arrow)

pattern of hepatic deposits and the inability to differentiate between post-therapeutic viable and non-viable malignant lesions. According to previous studies PET/ CT has showed better sensitivity and specificity for detection of hepatic and extra-hepatic metastasis than
CECT [12, 13]. In some studies, the sensitivity of $\mathrm{PET} / \mathrm{CT}$ for hepatic lesions was similar to that of MRI $[9,14]$. The final diagnosis of distant metastasis and/or local recurrence by PET/CT in posttherapeutic CRC in our study based on lesions

Table 3 Agreement of PET-CT with the reference modalities in detection of post-therapeutic colon cancer recurrence and metastasis (lesion-based analysis $n=252$ ) crosstab

\begin{tabular}{|c|c|c|c|c|c|}
\hline & & & \multicolumn{2}{|l|}{ Reference } & \multirow[t]{2}{*}{ Total } \\
\hline & & & Negative & Positive & \\
\hline \multirow[t]{4}{*}{ PET-CT result } & Negative & Number of Patients & 64 & 8 & 72 \\
\hline & & $\%$ & $91.4 \%$ & $4.4 \%$ & $28.6 \%$ \\
\hline & Positive & Number of Patients & 6 & 174 & 180 \\
\hline & & $\%$ & $8.6 \%$ & $95.6 \%$ & $71.4 \%$ \\
\hline \multirow[t]{2}{*}{ Total } & & Number of Patients & 70 & 182 & 252 \\
\hline & & $\%$ & $100.0 \%$ & $100.0 \%$ & $100.0 \%$ \\
\hline
\end{tabular}


Table 4 Agreement of CECT with the reference modalities in detection of post-therapeutic colon cancer recurrence and metastasis (lesion-based analysis $n=252$ ) crosstab

\begin{tabular}{|c|c|c|c|c|c|}
\hline & & & \multicolumn{2}{|l|}{ Reference } & \multirow[t]{2}{*}{ Total } \\
\hline & & & Negative & Positive & \\
\hline \multirow[t]{4}{*}{$\overline{C E-C T}$ result } & Negative & Number of Patients & 34 & 68 & 102 \\
\hline & & $\%$ & $48.6 \%$ & $37.4 \%$ & $40.5 \%$ \\
\hline & Positive & Number of Patients & 36 & 114 & 150 \\
\hline & & $\%$ & $51.4 \%$ & $62.6 \%$ & $59.5 \%$ \\
\hline \multirow[t]{2}{*}{ Total } & & Number of Patients & 70 & 182 & 252 \\
\hline & & $\%$ & $100.0 \%$ & $100.0 \%$ & $100.0 \%$ \\
\hline
\end{tabular}

analysis was evident in $70 \%$ of our patient population with sensitivity of $95.6 \%$, specificity of $91.4 \%$, NPV of $88.9 \%$, PPV of $96.7 \%$, and diagnostic efficacy of $94.4 \%$ compared with $62.6 \%, 48.6 \%, 76 \%, 33.3$ and $58 \%$ for CECT respectively. It showed a great role in the detection of small sized LNs, local operative bed recurrence, small suprarenal metastasis, early osseous deposits, and post-therapeutic evaluation of viable and non-viable malignant lesions (post-chemotherapy, post-radiotherapy). These results were quite similar to those obtained by Metser et al. [15] whose study included 158 post-operative colorectal carcinoma patients who presented with increasing CEA levels and equivocal explanation of these elevated levels by conventional imaging modalities. He reported sensitivities of PET/CT and MDCT of $98.1 \%$ and $66.7 \%$, and specificities of $75 \%$ and $62.5 \%$ respectively. The specificity in Metser et al. study for PET/CT was higher than that by Mittal et al. [16] in which he analyzed 73 post-operative CRC patients grouped into various categories depending on CEA level. PET/CT showed recurrences in $71 \%$ of CRC patients, CT showed recurrence in $23(45 \%)$ patients, and the positive rate increased with the CEA level. The study results by Choi et al. [17] to assess the value of 18F-FDG PET/ CT in detecting local or distant recurrence in 269 post-operative CRC patients and to compare its accuracy with conventional imaging studies, showed overall sensitivity, specificity, accuracy, PPV, and NPV of $94.7 \%, 96.0 \%, 95.8 \%, 78.2 \%$, and $99.2 \%$ for PET/CT, and $86.8 \%, 97.6 \%, 96.2 \%, 84.6 \%$, and $98.0 \%$ for conventional imaging studies, respectively. As reported previously and confirmed by our study, Chiewvit

Table 5 Diagnostic performance of PET/CT in comparison with CECT by regional lesion-based analysis

\begin{tabular}{llllll}
\hline Sensitivity & Specificity & $\begin{array}{l}\text { Positive } \\
\text { predictive } \\
\text { value }\end{array}$ & $\begin{array}{l}\text { Negative } \\
\text { predictive } \\
\text { value }\end{array}$ & $\begin{array}{l}\text { Diagnostic } \\
\text { efficacy }\end{array}$ \\
\hline PET-CT & $95.6 \%$ & $91.4 \%$ & $96.7 \%$ & $88.9 \%$ & $94.4 \%$ \\
CECT & $62.6 \%$ & $48.6 \%$ & $76.0 \%$ & $33.3 \%$ & $58 \%$ \\
\hline
\end{tabular}

et al. [13] have shown that 18F-FDG PET/CT is a useful method in post-operative evaluation of patients with suspected recurrent colorectal cancerous lesions and a normal CEA level. When conventional imaging methods have shown equivocal findings, 18F-FDG $\mathrm{PET} / \mathrm{CT}$ is effective and helpful to distinguish local recurrences or metastases from post-operative changes or benign disease findings that may not be meaningful. The sensitivity of FDG-PET imaging for detection of mucinous carcinoma was proven by many authors to be significantly lower than that for nonmucinous carcinomas owing to little or negligible uptake of the tracer [3]. In our study, mucinous adenocarcinoma metastases constituted the main factor responsible for false negative scans Therefore, it was suggested that for mucinous adenocarcinoma patients with negative FDG-PET imaging results, other imaging modalities should be recommended for further diagnosis [11, 18]. Limitations of this study were as follows: (1) the sensitivity, specificity, and PPV of PET/CT may be overestimated because the study population included only those patients with suspected local recurrence or metastasis by regular follow-up program and most (87\%) of them have elevated serum CEA levels. According to Zhang et al. (11) the sensitivity, specificity, and accuracy of FDG PET/CT were $81.3 \%, 80 \%$, and $80.6 \%$ in patients with normal CEA and equivocal or meaningless findings at conventional imaging modalities. (2) Histopathologic confirmation was lacking in 28 patients.

\section{Conclusion}

In conclusion, PET/CT proved to be a better method to evaluate post-therapeutic colorectal cancer patients. It detected viable residual tumor cells in operative bed scar, small LNs, hepatic focal lesions, peritoneal deposits, pulmonary masses, bone deposits, and suprarenal deposits with significantly higher specificity and sensitivity than CECT avoiding unnecessary surgeries

\section{Abbreviations}

18F-FDG: 18 Fluorine fluorodeoxyglucose; CEA: Carcinoembryonic antigen; CECT: Contrast-enhanced computerized tomography; CRC: Colorectal cancer; MRI: Magnetic resonance imaging; PET/CT: Positron emission tomography; SUVmax: Standardized uptake value

\section{Acknowledgements}

The scientific guarantor of this publication is R.H.B. The authors of this manuscript declare no relationships with any companies whose products or services may be related to the subject matter of the article. No complex statistical methods were necessary for this paper.

\section{Authors' contributions}

Corresponding author RHB contributed in the acquisition analysis and interpretation of the imaging studies, has drafted the manuscript, and revised and approved the submitted version and agreed to be personally accountable for the author's own contributions and to ensure that questions related to the accuracy or integrity of any part of the work, even ones in which the author was not personally involved, are appropriately investigated, 
resolved, and the resolution documented in the literature. First author NAMC provided the main idea and the main design of the word; helped in collection of the clinical and pathologic data; contributed in the acquisition, analysis, and interpretation of the imaging studies; and revised and approved the submitted version. Third author MABS collected the clinical and pathologic data, contributed in the acquisition of the quantitative measures, contributed to drafting of the manuscript, and revised and approved the submitted version. All authors have read and approved the manuscript.

\section{Funding}

The authors state that this work has not received any funding. Institutional Review Board approval was obtained.

\section{Availability of data and materials}

All datasets used and/or analyzed during the current study are available in the radiology department of AL Maadi Egyptian military Hospital.

\section{Ethics approval and consent to participate}

The study was approved by the ethical committee of Al Maadi Egyptian military hospital on January 2014 (no reference number was given at that time). Written consent for participation in the study was obtained from all patients or relatives of patients.

\section{Consent for publication}

Written informed consent for publication of the study was obtained from the patients themselves.

\section{Competing interests}

This statement is to certify that all authors have seen and approved the manuscript being submitted. We warrant that the article is the authors' original work. We warrant that the article has not received prior publication and is not under consideration for publication elsewhere. On behalf of all co-authors, the corresponding author shall bear full responsibility for the submission.

\section{Author details}

${ }^{1}$ Ain Shams University, Radiodiagnosis Department, Cairo, Egypt. ${ }^{2}$ Egyptian Military Hospital, Cairo, Egypt.

Received: 20 November 2019 Accepted: 26 November 2019

Published online: 07 January 2020

\section{References}

1. Fatima AH, Robin PB (2009) Colorectal cancer epidemiology: incidence, mortality, survival, and risk factors. Clin Colon Rectal Surg 22(4):191-197

2. Ozkan E, Soydal C, Araz M, Kir KM, Ibis E (2012) The role of 18F-FDG PET/CT in detecting colorectal cancer recurrence in patients with elevated CEA levels. Nucl Med Commun. 33:395-402

3. Borasio P, Gisabella M, Billé A, Righi L, Longo M, Tampellini M, Ardissone F (2011) Role of surgical resection in colorectal lung metastases: analysis of 137 patients. Int J Colorectal Dis. 26:183-190

4. Maas M, Rutten IJ, Nelemans PJ, Lambregts DM, Cappendijk VC, Beets GL, Beets-Tan RG (2011) What is the most accurate whole-body imaging modality for assessment of local and distant recurrent disease in colorectal cancer? A metaanalysis: imaging for recurrent colorectal cancer. Eur J Nucl Med Mol Imaging. 38:1560-1571

5. Gan S, Wilson K, Hollington P (2007) Surveilance of patients following surgery with curative intent for colorectal cancer. World J Gastronterol 13: 3816-3823

6. Tan E, Gouvas N, Nicholls RJ, Ziprin P, Xynos E, Tekkis PP (2009) Diagnostic precision of carcinoembryonic antigen in the detection of recurrence of colorectal cancer. Surg Oncol. 18:15-24 [PubMed]

7. Israel O, Kuten A (2007) Early detection of cancer recurrence: 18F-FDGPET/ $\mathrm{CT}$ can make a difference in diagnosis and patient care. Nuclear Medicine 48:28-35

8. Israel O, Mor M, Guralnik L (2004) Is 18F-FDG PET/CT useful for imaging and management of patients with suspected occult recurrence of cancer? J Nucl Med 45:2045-2051

9. Niekel MC, Bipat S, Stoker J (2010) Diagnostic imaging of colorectal liver metastases with CT, MR imaging, FDG PET, and/or FDG PET/CT: a meta- analysis of prospective studies including patients who have not previously undergone treatment. Radiology. 257:674-684

10. Fiocchi F, lotti V, Ligabue G, Malavasi N, Luppi G, Bagni B, Torricelli P (2011) Role of carcinoembryonic antigen, magnetic resonance imaging, and positron emission tomography-computed tomography in the evaluation of patients with suspected local recurrence of colorectal cancer. Clin Imaging. 35:266-273

11. Zhang $Y$, Feng $B$, Zhang G-L, Hu M, Zheng F, Zhao F, Zhang $X-L$, Kong $L$, Jin-Ming Y (2014) Value of 18F-FDG PET-CT in surveillance of postoperative colorectal cancer patients with various carcinoembryonic antigen concentrations. World J Gastroenterol. 20(21):6608-6614

12. Shamim SA, Kumar R, Halanaik D, Shandal V, Reddy RM, Bal CS, Malhotra A (2010) Role of FDG-PET/CT in detection of recurrent disease in colorectal cancer. Nucl Med Commun. 31:590-596

13. Chiewvit $\mathrm{S}$, Jiranantanakorn $\mathrm{T}$, Apisarnthanarak $\mathrm{P}$, Kanchaanapiboon $\mathrm{P}$ Hannanthawiwat C, Ubolnuch K, Phongsawat N, Chiewvit P (2013) Detection of recurrent colorectal cancer by 18F-FDG PET/CT comparison with contrast enhanced CT scan. J Med Assoc Thai. 96:703-708

14. Patel S, McCall M, Ohinmaa A, Bigam D, Dryden DM (2011) Positron emission tomography/computed tomographic scans compared to computed tomographic scans for detecting colorectal liver metastases: a systematic review. Ann Surg. 253:666-671

15. Metser U, You J, McSweeney S, Freeman M, Hendler A (2010) Assessment of tumor recurrence in patients with colorectal cancer and elevated carcinoembryonic antigen level: FDG PET/CT versus contrast-enhanced 64MDCT of the chest and abdomen. AJR Am J Roentgenol 194:766-771

16. Mittal BR, Senthil R, Kashyap R, Bhattacharya A, Singh B, Kapoor R, Gupta R (2011) 18F-FDG PET-CT in evaluation of postoperative colorectal cancer patients with rising CEA level. Nucl Med Commun 32(9):789-793

17. Choi EK, Yoo LR, Han EJ, Oo JH, Kim SH, Chung SK (2010) Value of surveillance F-18 FDG PET/CT in colorectal cancer: comparison with conventional imaging studies. J Nucl Med 51:1208

18. Fitzmaurice C, Allen C et al (2017) Global, regional, and national cancer incidence, mortality, years of life lost, years lived with disability, and disability-adjusted life-years for 32 cancer groups, 1990 to 2015: a systematic analysis for the global burden of disease study. JAMA Oncol 3:524

\section{Publisher's Note}

Springer Nature remains neutral with regard to jurisdictional claims in published maps and institutional affiliations.

\section{Submit your manuscript to a SpringerOpen ${ }^{\circ}$ journal and benefit from:}

- Convenient online submission

- Rigorous peer review

- Open access: articles freely available online

High visibility within the field

- Retaining the copyright to your article

Submit your next manuscript at $\boldsymbol{\nabla}$ springeropen.com 